\title{
Extrarenal effects on the pathogenesis and relapse of idiopathic nephrotic syndrome in Buffalo/Mna rats
}

\author{
Ludmilla Le Berre, ${ }^{1}$ Yann Godfrin, ${ }^{1}$ Eberhard Günther, ${ }^{2}$ Françoise Buzelin, ${ }^{3}$ \\ Sabine Perretto, ${ }^{1}$ Helga Smit, ${ }^{1}$ Dontscho Kerjaschki, ${ }^{4}$ Claire Usal, ${ }^{1}$ Cristina Cuturi, ${ }^{1}$ \\ Jean-Paul Soulillou, ${ }^{1}$ and Jacques Dantal ${ }^{1}$
}

\begin{abstract}
${ }^{1}$ Institut National de la Santé et de la Recherche Médicale (INSERM), Immunointervention dans les Allo et Xenotransplantations, and Institut de Transplantation et Recherche en Transplantation (ITERT), Nantes, France

${ }^{2}$ Abteilung Immungenetik, Georg-August-Universität Göttingen, Göttingen, Germany

${ }^{3}$ Service d' Anatomopathologie, Centre Hospitalier Universitaire Hotel Dieu, Nantes, France

${ }^{4}$ Institut für Klinische Pathologie, Vienna, Austria
\end{abstract}

Address correspondence to: Jean-Paul Soulillou, INSERM 437, 30 Boulevard Jean Monnet, 44093 Nantes, France. Phone: 33-2-40-08-74-10; Fax: 33-2-40-08-74-11; E-mail: jps@nantes.inserm.fr.

Received for publication April 2, 2001, and accepted in revised form December 18, 2001.

\begin{abstract}
Buffalo/Mna rats spontaneously develop a focal segmental glomerulosclerosis with a histological pattern similar to the human disease. In this study, we investigated the potential of recurrence of the disease by transplantation of normal kidneys into Buffalo/Mna recipients. Kidneys from healthy LEW.1W rats were grafted into proteinuric 6-month-old Buffalo/Mna rats without or with specific tolerance induction following donor-specific transfusion (DST) aimed at controlling host anti-donor immune responses. The inverse combination was carried out to determine whether a proteinuric Buffalo/Mna kidney can recover its permselectivity in a normal environment. As a control, LEW.1W kidneys were grafted into Wistar Furth recipients. After transplantation without DST, recurrence of proteinuria in LEW.1W kidneys appeared at approximately 10 days, possibly associated with rejection of the graft. In the same combination with DST, proteinuria occurred after 20 days, and the attendant glomerular damage suggested that the initial kidney disease had recurred. Transplanted control animals remained free of proteinuria. In the opposite combination, the proteinuria and the lesions of Buffalo/Mna kidneys regressed after transplantation into healthy LEW.1W rats. The recurrence of proteinuria after transplantation in Buffalo/Mna and the remission of lesions in Buffalo/Mna kidneys transplanted into normal hosts suggests that Buffalo/Mna rats express circulating albuminuric factors, which may be relevant to the relapse of idiopathic nephrotic syndrome in humans.
\end{abstract}

J. Clin. Invest. 109:491-498 (2002). DOI:10.1172/JCI200212858.

\section{Introduction}

Idiopathic nephrotic syndrome (INS) and primary focal segmental glomerulosclerosis (FSGS) are diseases of unknown etiology. Albuminuria and renal biopsy examination showing a hyalinosis following synechia between the glomerulus and its capsule are necessary for diagnosis. Most patients (70-80\%) with INS are sensitive to unspecific anti-inflammatory and/or immunosuppressive treatments such as corticoids, cyclosporine $\mathrm{A}$, and cyclophosphamide (for review see ref. 1), but about $20 \%$ ultimately require hemodialysis for end-stage renal failure and eventually need a kidney transplant (2). Unfortunately, $25-40 \%$ of patients who receive transplants rapidly suffer from a relapse of the initial disease on their graft, which in approximately half of transplant patients leads to graft loss (3). In 90\% of these patients, FSGS recurrence manifests itself immediately after the transplantation, strongly suggestive of the presence of an albuminuric (proteinuric) plasmatic factor(s), a hypothesis strengthened by the beneficial effect of plasmatic exchanges (4-6). In addi- tion, we have demonstrated that major immunoglobulin depletion by extracorporeal adsorption onto two different columns, protein A (7) and sheep anti-human Ig (8), dramatically decreased proteinuria, suggesting that Ig could act as a carrier for the factor. However, the nature of this proteinuric factor and its mechanism of action in these relapses remains mysterious. There is therefore an urgent need for a better understanding of the mechanisms that lead to FSGS and its relapse. Several attempts have been made to trace a putative proteinuric activity that alters the glomerular albumin permselectivity in patients with relapsing disease (9-11). Despite these efforts, no convincing experimental link between activity detected in in vitro and in vivo physiological models has been shown (12). Therefore, the nature and role of these in vitro activities in disease etiology remain uncertain. Some data have suggested that Ig-free fractions can cause both altered glomerular permeability in vitro and trigger proteinuria in the rat; however, fractions from non-FSGS nephrotic syndrome were not tested (13). 


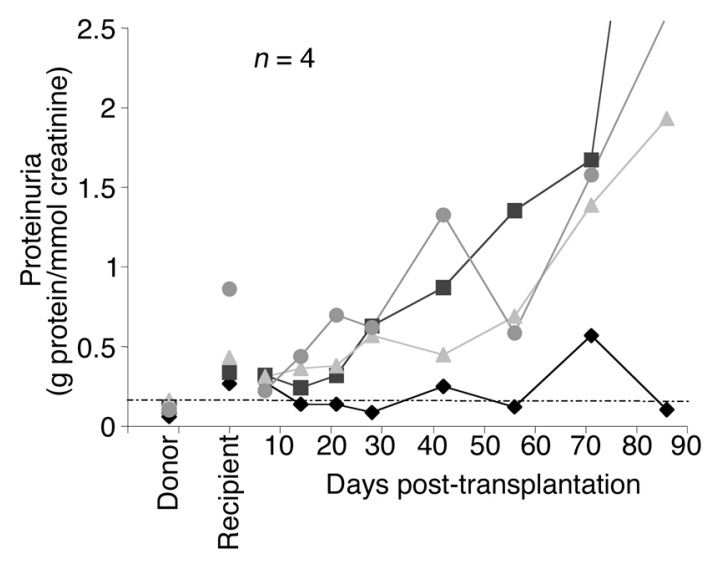

Figure 1

Proteinuria following renal transplantation of nonproteinuric kidneys from LEW.1W rats into proteinuric binephrectomized Buffalo/Mna recipients. Proteinuria is expressed as the ratio of the urinary protein to urinary creatinine concentration. Four animals were transplanted without any immunosuppressive treatment (or DST).

A spontaneous animal model where renal lesions resemble idiopathic human FSGS may provide a promising tool to elucidate FSGS pathogenesis and to test the effect of new drugs on proteinuria. Several experimental models of human FSGS-like glomerular lesions have been described, including aging-associated nephropathy (14), nephrotic reduction (15-16), and puromycin or aminonucleoside toxic-induced nephrosis (17-19). However, even though these models mimic the human glomerular lesions and may help to identify the mechanisms involved, they are of limited value in terms of the characterization of the putative albuminuric factor(s) that could be instrumental in FSGS pathogenesis. In 1983, Kato et al. (20) showed that the Buffalo/Mna rats spontaneously develop lesions mimicking those observed in human idiopathic FSGS with, at 2 months of age, epithelial cell alterations with foot process flattening and vacuolization at the ultrastructural level (21). In addition, these animals are normotensive and are not uremic. Furthermore, we have shown that the proteinuria in these animals is, to some extent, sensitive to corticosteroids, cyclosporine A, and cyclophosphamide (Le Berre et al., personal communication). The possible involvement of plasmatic factors and the ability of the disease to recur in a normal kidney after transplantation have not yet been explored in this model.

Ideal models of human diseases are rare. Of course, whether the Buffalo/Mna rat offers a relevant model of a disease of unknown etiology, especially INS, is impossible to predict precisely. However, in this study we demonstrate, we believe for the first time, the recurrence of proteinuria in binephrectomized Buffalo/Mna rats after transplantation with kidneys from healthy rats. In addition, we show that proteinuria, as well as renal lesions, from Buffalo/Mna kidneys decreases when proteinuric kidneys are transplanted into normal recipients. Thus, our study provides a new model that has some similarities to the human disease and that may be useful for the understanding of its mechanisms.

\section{Methods}

Animals. The Buffalo/Mna rat line, maintained in our laboratory, was originally kindly provided by S. Saito (Central Experimental Institute, Nokawa, Kawasaki, Japan). All animals were born from a unique couple and bred for at least ten generations. Despite these animals being named Buffalo and thus being supposed to carry the RT $1^{\mathrm{b}}$ haplotypes, some additional tests were performed in our laboratory. The animals were typed by serology using Serotec mAb's (Argene SA, Varilhes, France) and found to be positive for anti-RT1 ${ }^{\mathrm{u}} \mathrm{Ab}$ 's. Subsequently, mixed lymphocyte cultures (MLCs) were performed using Buffalo/Mna-responding cells and a panel of RT1-typed stimulating cells. Buffalo/Mna MLCs only gave a negative reaction against $\mathrm{RT} 1^{\mathrm{u}}$-stimulating cells. Thus, we considered that Buffalo/Mna rats were RT1 ${ }^{\mathrm{u}}$. Next, in the absence of published genotyping, we determined the genotype of the Buffalo/Mna rat. The sequencing of the RT1-Ba and the RT1-A1 exon 2 demonstrated that both the $\mathrm{u}$ and $\mathrm{b}$ haplotypes were present in this strain. The inbred LEW.1W (RT1 $\left.{ }^{\mathrm{u}}\right)$ and Wistar Furth $\left(\mathrm{RT} 1^{\mathrm{u}}\right)$ rats were obtained from an established colony (Janvier, Le Genest Saint Isle, France). All animals were between 4 and 6 months old and were fed with standard laboratory food. The animal care involved in this study was in strict accordance with the institutional guidelines.

Proteinuria measurement. The animals were placed in metabolic cages for 24 hours before measurement, with free access to water but without food pellets that could fall into the urine collector and bias the measurement of urinary proteins. The total urinary protein concentration (grams per liter) was measured by a colorimetric

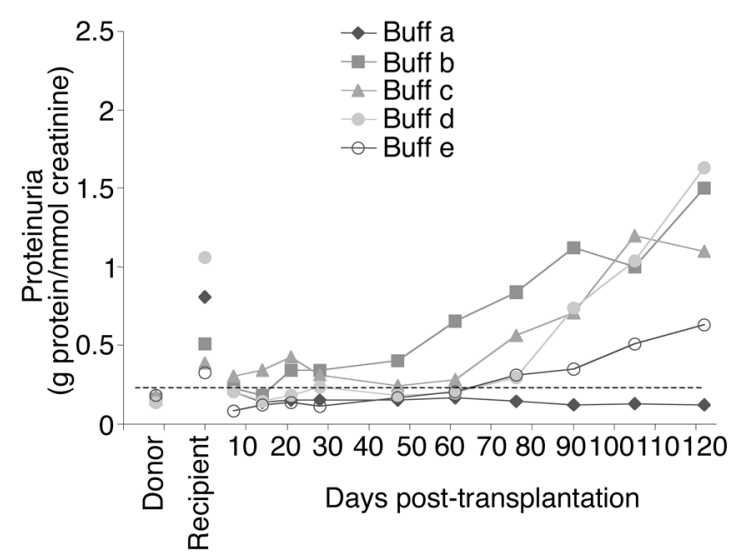

Figure 2

Proteinuria following renal transplantation of nonproteinuric kidneys from LEW.1W rats into proteinuric binephrectomized Buffalo/Mna recipients treated with DST before transplantation to induce tolerance. Proteinuria is expressed as the ratio of the urinary protein to urinary creatinine concentration. Five animals were transplanted. Buff, Buffalo/Mna. 

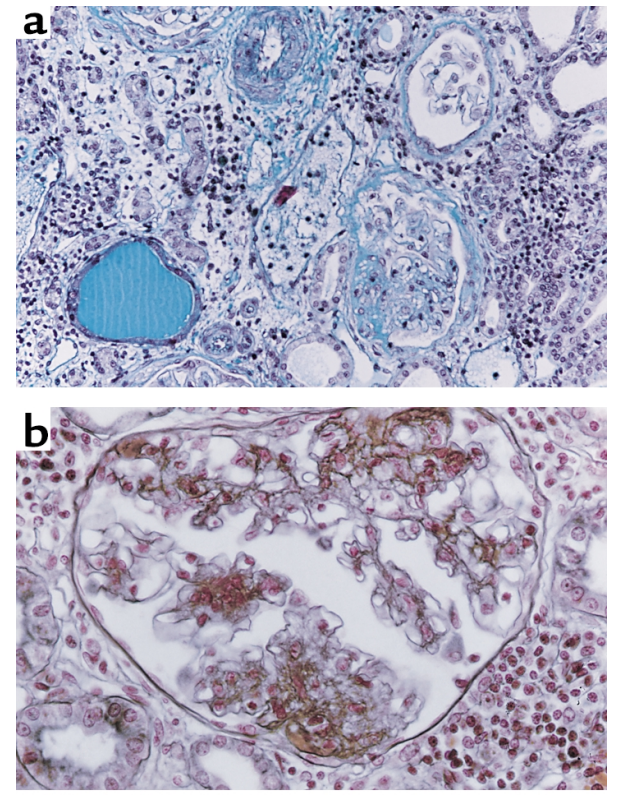

\section{Figure 3}

Light microscope examination of LEW.1W kidneys transplanted into proteinuric Buffalo/Mna rat recipients that have received a tolerizing (DST) regimen. (a) Masson's trichrome staining $(\times 200)$ shows a moderate interstitial infiltrate with severe tubular alterations. (b)The arteries remained normal and the glomeruli showed typical FSGS lesions (Jones staining, $\times 400)$.

method using a Hitachi autoanalyser (RocheBoehringer, Meylan, France). Urinary creatinine (millimoles per liter) was measured by the Jaffé method. Proteinuria (grams per millimole) was expressed according to the following formula: proteinuria = urinary proteins/urinary creatinine. It was considered abnormal when the value was greater than $0.2 \mathrm{~g} / \mathrm{mmol}$.

Renal transplantation. Healthy rat kidneys (LEW.1W) were transplanted into proteinuric Buffalo/Mna recipients, and Buffalo/Mna rat kidneys were also placed into nonproteinuric LEW. $1 \mathrm{~W}$ recipients. Rats were anesthetized for approximately 90 minutes by ethylether inhalation. The surgical technique has been described elsewhere (22). The first nephrectomy was performed during the transplantation procedure and the second nephrectomy 7 days later. In the first series of experiments, animals were grafted without any other procedure because donor and recipient haplotypes were compatible according to the MLC test (see above). In a second series, animals received donor-specific transfusions (DST) (1 ml of donor blood injected intravenously 14 and 7 days before transplantation) to induce tolerance (23-25). In a third series, an inverse experiment aimed at exploring whether proteinuria of a diseased kidney could disappear after transplantation into a normal environment was performed. Kidneys from proteinuric Buffalo/Mna (i.e., $>0.2 \mathrm{~g} / \mathrm{mmol}$ ) were thus grafted into nonproteinuric LEW.1W rats following the same DST protocol. As controls, kidneys from LEW.1W rats $\left(\mathrm{RT} 1^{\mathrm{u}}\right)$ were transplanted into Wistar
Furth rats $\left(\mathrm{RT} 1^{\mathrm{u}}\right)$; neither strain was proteinuric (i.e., $<0.17 \mathrm{~g} / \mathrm{mmol}$ ). Finally, additional controls were obtained by monitoring the proteinuria of agematched unmodified and uninephrectomized proteinuric Buffalo/Mna rats. Blood creatinine and proteinuria were regularly measured in the transplanted animals until sacrifice.

Light and electron microscope examination. LEW.1W kidney samples grafted into Buffalo/Mna recipients were obtained at the time of sacrifice: day 86 for the first set of experiments (non-DST group) and day 45, 80, and 122 for both the DST group and the control group (LEW.1W into Wistar Furth rats). The Buffalo/Mna kidneys transplanted into LEW.1W recipients were harvested at day 80 , the time of sacrifice. Tissues were fixed for 20 minutes in Carnoy solution and then in 10\% buffered formalin and embedded in paraffin. Threemicrometer sections were stained with hematoxylin and eosin, periodic acid-Schiff (PAS), Masson's trichrome, and periodic acid-silver methenamine. All histological parameters (interstitial infiltrate, tubule casts, tubular distension, percentage of segmental lesions, destroyed flocculi, and injured glomeruli) were analyzed in a blinded fashion. Finally, to quantify the level of graft rejection, we employed the Banff classification described by Solez et al. (26), whereby inflammatory responses in four different kidney compartments (interstitial, tubular, glomerular, and vascular)
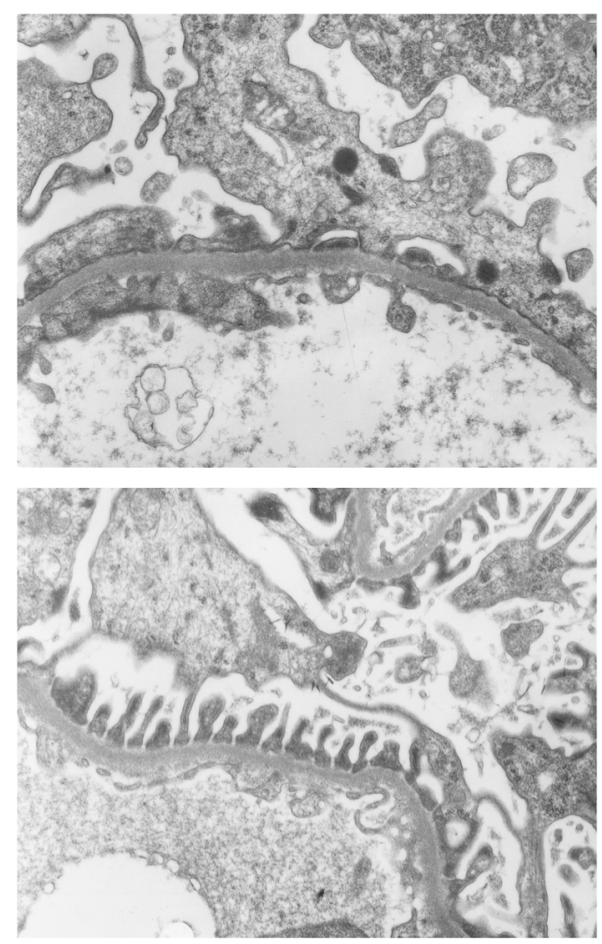

\section{Figure 4}

Electron microscopic examination of glomeruli of (a) LEW.1W kidneys grafted into Buffalo/Mna rats $(\times 10,000)$ and glomeruli of $(\mathbf{b})$ LEW.1W kidneys transplanted into Wistar Furth (WF) rats $(\times 10,000)$. (a) A substantial fusion of the foot processes and a moderate subendothelial edema. (b) A normal histological pattern without foot process fusion. 
Table 1

Summary of the light microscopic examination of the two groups of recipients treated with DST before transplantation

\begin{tabular}{|c|c|c|c|c|c|c|c|c|c|c|}
\hline \multirow{2}{*}{$\begin{array}{l}\text { Donor/recip. } \\
\text { strain }\end{array}$} & \multirow{2}{*}{$\begin{array}{c}\text { Interstitial } \\
\text { infiltrate }\end{array}$} & \multicolumn{3}{|c|}{ Tubular lesion } & \multicolumn{3}{|c|}{ Glomerular lesion } & \multirow[b]{2}{*}{ BANFF } & \\
\hline & & $\begin{array}{c}\text { Neutrophilic } \\
\text { casts }\end{array}$ & $\begin{array}{c}\text { Eosinophilic } \\
\text { casts }\end{array}$ & Dilatation & $\begin{array}{c}\% \\
\text { Segmental } \\
\text { injury }\end{array}$ & $\begin{array}{c}\% \\
\text { Destroyed } \\
\text { flocculus }\end{array}$ & $\begin{array}{c}\% \\
\text { Foot process } \\
\text { fusion }\end{array}$ & & $\begin{array}{c}\text { Proteinuria } \\
(\mathrm{g} / \mathrm{mmol}) \\
\text { at sacrifice }\end{array}$ & $\begin{array}{c}\text { S creatinine } \\
(\mu \mathrm{mol}) \\
\text { at sacrifice }\end{array}$ \\
\hline Lew.1w/WF (1) & + & - & - & - & 10 & 0 & 5 & i0 t0 g0 vo aho & 0.31 & 52 \\
\hline Lew.1w/WF (2) & + & - & - & - & 23 & 0 & 10 & i0 t0 g0 vo aho & 0.19 & 55 \\
\hline Lew.1w/WF (3) & + & - & - & - & 7 & 0 & 10 & i0 t0 g0 vo aho & 0.3 & 40 \\
\hline Lew.1w/WF (4) & + & - & - & - & 17 & 0 & 10 & i0 t0 g0 vo aho & 0.72 & 50 \\
\hline Mean \pm SE & & & & & $14 \pm 4$ & 0 & $9 \pm 2.5$ & & $0.38 \pm 0.11$ & $49 \pm 3.2$ \\
\hline Lew.1w/Buf (a) & ++ & - & - & - & 0 & 0 & 0 & i2 t1 g1 vo aho & 0.12 & 43 \\
\hline Lew.1w/Buf (b) & +++ & + & ++ & +++ & 30 & 10 & 40 & i2 t1 g1 vo aho & 1.5 & 165 \\
\hline Lew.1w/Buf (c) & +++ & + & ++ & +++ & 13 & 13 & 20 & i2 t1 g1 vo aho & 0.94 & 152 \\
\hline Lew.1w/Buf (d) & +++ & - & + & +++ & 23 & 0 & 10 & i2 t1 g1 vo aho & 1.63 & 63 \\
\hline Lew.1w/Buf (e) & + & - & + & ++ & 13 & 2 & ND & i1 t0 g1 v0 ah0 & 0.63 & 46 \\
\hline Mean \pm SE & & & & & $15.8 \pm 3$ & $5 \pm 3$ & $17.5 \pm 3$ & & $0.97 \pm 0.62$ & $293.8 \pm 59$ \\
\hline
\end{tabular}

The first group is represented by the analysis of LEW.1W kidneys transplanted into Wistar Furth $(n=4)$ rats and the second into Buffalo/Mna $(n=5)$ rats. WF, Wistar Furth. Recip., recipient.

are judged at the histological level, and each receive a score from 0 to 3 . For electron microscope examination, kidney biopsies were fixed in $2.5 \%$ glutaraldehyde, postfixed in a mixture of $2 \%$ osmium tetroxide in 200 $\mathrm{mM}$ cacodylate buffer, $\mathrm{pH} 7.2$, and embedded in Epon 815. Thin sections were stained with lead citrate and uranyl acetate and examined using a Hitachi electron microscope at $2,000 \mathrm{KeV}$.

Immunofluorescence. Kidney samples were embedded in optimal cutting tissue compound (Tissue-Tek, Miles Inc., Elkhart, Indiana, USA), snap-frozen in precooled isopenthane, and stored at $-80^{\circ} \mathrm{C}$ until use. Frozen $6-\mu \mathrm{m}$ tissue sections were stained with affinity-purified FITC-conjugated mouse $\mathrm{F}\left(\mathrm{ab}^{\prime}\right)_{2}$ anti-rat IgG and with FITC-conjugated goat anti-rat IgM (The Jackson Laboratories, West Grove, Pennsylvania, USA). Analysis was performed using an Olympus ultraviolet microscope.

Statistical analysis. The nonparametric Mann-Whitney test was used to compare proteinuria values between groups for each point, and the paired $t$ test was used to compare the proteinuria at different days in the same group. $P$ values less than 0.05 were considered to be significant.

\section{Results}

Proteinuria relapses in binephrectomized Buffalo/Mna recipients of normal LEW.1W kidneys

LEW.1W kidney transplantation into nephrectomized Buffalo/Mna recipients. On the basis of negative MLC, a first series of transplants was performed without a tolerance-inducing regimen or immunosuppressive treatment. Figure 1 shows a progressive increase in proteinuria in three of the four Buffalo/Mna rat recipients of healthy LEW.1W kidneys, with a mean proteinuria above $0.2 \mathrm{~g} / \mathrm{mmol}$ after 10 days. Blood creatinine levels remained stable $(121.5 \pm 69 \mu \mathrm{mol} / \mathrm{l})$ after the transplantation. However, when omitting one animal that neither developed proteinuria nor glomerular injury, the histological study showed a mixture of glomerular and tubular lesions that did not allow for an unambiguous distinction between either recurrence of a primary glomerulopathy or a secondary FSGS due to chronic rejection. Indeed, despite the fact that no histologically obvious lesions of chronic rejection were observed, significant fibrosis and interstitium infiltration occurred, making it impossible to conclude that the pattern observed only represented recurrence of the primary glomerulopathy. Podocyte injury, measured in terms of foot process fusion, was assessed on the day of sacrifice and found to affect between $20 \%$ and $40 \%$ of the glomeruli.

To clarify some difficulties encountered in the interpretation of histological lesions, a second series of experiments was performed following a toleranceinducing regimen. In comparison with non-DSTtreated Buffalo/Mna recipients, proteinuria relapse was delayed and reached the threshold of $0.2 \mathrm{~g} / \mathrm{mmol}$ 20 days after transplantation (Figure 2 ). One rat (a, in Table 1) did not develop any proteinuria or glomerular lesions even 122 days after the transplantation. Without this animal, which did not present proteinuria and showed few histological lesions, proteinuria of LEW.1W to Buffalo/Mna group was significantly different from the control group $(P<0.05)$. Serum creatinine levels remained stable $(93.8 \pm 59 \mu \mathrm{mol})$ after transplantation and not significantly different from the control group ( $P=$ not significant). Histological analysis of the LEW.1W to Buffalo/Mna combination group (Table 1) showed a substantial interstitial infiltrate, a low number of granular and eosinophilic casts, a high level of tubular dilatation, and segmental 


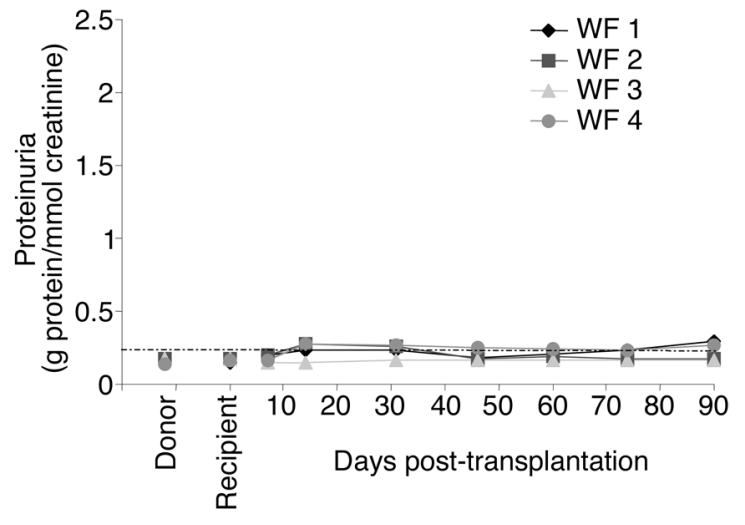

Figure 5

Proteinuria following renal transplantation of nonproteinuric kidneys from LEW.1W rats into nonproteinuric, binephrectomized Wistar Furth recipients treated with DST before transplantation to induce tolerance. Proteinuria is expressed as the ratio of the urinary protein to urinary creatinine concentration. Four animals were transplanted. WF, Wistar Furth.

glomerulosclerotic lesions with some destroyed flocculi $(5 \% \pm 3 \%$ vs. $0 \%$ for the control group) (Figure 3 ). Moreover, when compared with the control group, foot process fusion was found in $17.5 \% \pm 3 \%$ of glomeruli, about threefold higher than in the control group $(9 \% \pm 2.5 \%)$ (Table 1). Glomerular mesangiolysis was also occasionally observed. A low Banff classification value ( 0 to IA), indicating some lymphocyte infiltration or areas of interstitial fibrosis, represents only weak evidence for rejection. Finally, vessels were found to be normal and did not exhibit any signs of chronic rejection and/or hypertension.

At day 45, when animals were weakly proteinuric, there was no significant difference in the glomerular and tubular pattern of LEW.1W kidneys transplanted into Buffalo/Mna rats as compared with the control group (LEW.1W into Wistar Furth) (data not shown). In the two groups, on day 80 , the histological pattern was similar to that observed on day 122 , but with less tubular and glomerular lesions. However, on day 80, electronic microscopy revealed more podocytic alterations in the LEW.1W kidneys grafted into Buffalo/Mna than in the control group (Figure 4).

LEW.1W kidney transplantation into binephrectomized Wistar Furth recipients. Since there are no Buffalo/Mna animals without proteinuria, control grafts were performed in other strains in an attempt to evaluate whether a minimal rejection process could induce proteinuria in recipients having received pregraft DST. Thus, Wistar Furth recipients received the same tolerance-inducing regimen as the Buffalo/Mna. Figure 5 shows that there was no increase in proteinuria in binephrectomized Wistar Furth recipients. Furthermore, no symptoms of rejection and no tubular injuries were noticed. Histological examination (Table 1) showed only minor interstitial infiltration, segmental sclerosis in less than $15 \%$ of glomeruli, and only $9 \% \pm 2.5 \%$ foot process fusion (Figure 6 ).
Proteinuria of Buffalo/Mna kidneys regresses in healthy binephrectomized LEW.1W recipients

The kidney transplantations were performed after tolerance induction following pregraft DST. Figure $7 \mathrm{a}$ shows a dramatic decrease in proteinuria of Buffalo/Mna kidneys transplanted into anephric LEW.1W rats. Moreover, proteinuria reverted to roughly normal values after transplantation, and renal function remained good and stable after transplantation and until sacrifice on day 80 (serum creatinine $=38$ and $29 \mu \mathrm{mol}$ ). At this time, histological lesions in Buffalo/Mna kidneys reverted to a subnormal renal pattern (Figure 7b). In contrast, in both age-matched unmodified and uninephrectomized Buffalo/Mna rats, proteinuria that developed showed an increase and was different from the transplanted group (Figure 7a).

Absence of significant Ig deposition in glomeruli of transplanted kidneys

To eliminate the possibility of a post-transplantation immune response against a glomerular constituent differing between Buffalo/Mna and LEW.1W strains that could have triggered the proteinuria, Ig deposits were studied in the transplanted kidneys.

No deposits of IgG were found in kidneys grafted into Buffalo/Mna rats or in control kidneys grafted into Wistar Furth rats. Irregular mesangial IgM staining was present at trace levels in LEW.1W kidneys grafted either into Buffalo/Mna recipients or into nonproteinuric recipients (Wistar Furth). In some glomeruli of LEW. $1 \mathrm{~W}$ rat kidneys grafted into
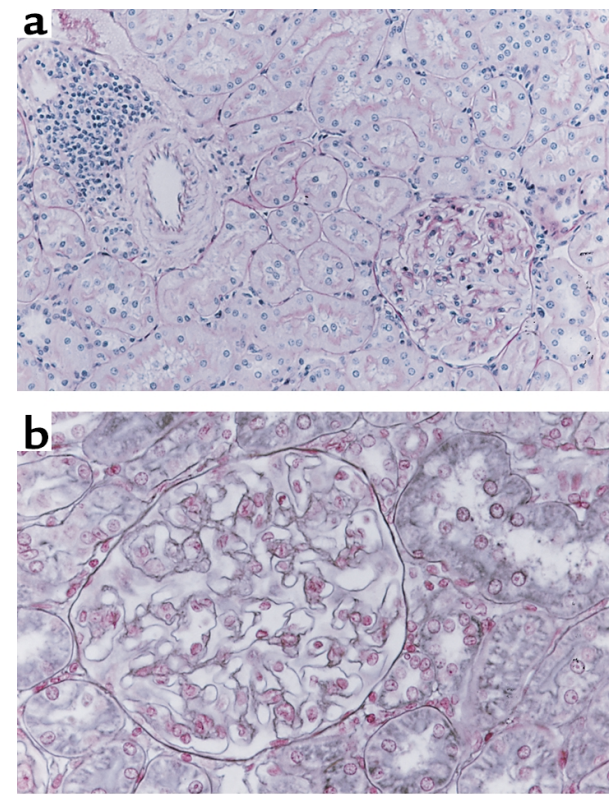

\section{Figure 6}

Light microscope examination of LEW.1W kidneys into nonproteinuric, DST-treated, Wistar Furth rats (control group). (a) PAS staining $(\times 200)$ shows a focal interstitial infiltrate without tubular alterations. (b) The glomeruli remained almost normal (Jones staining, $\times 400$ ). 


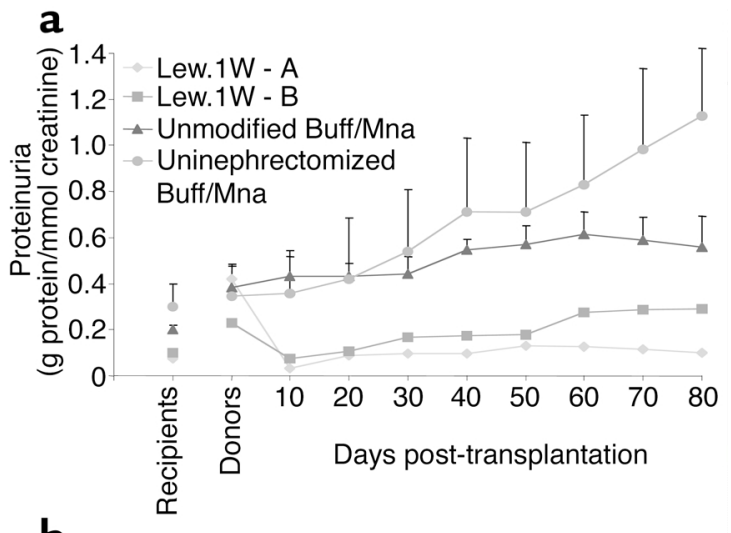

b

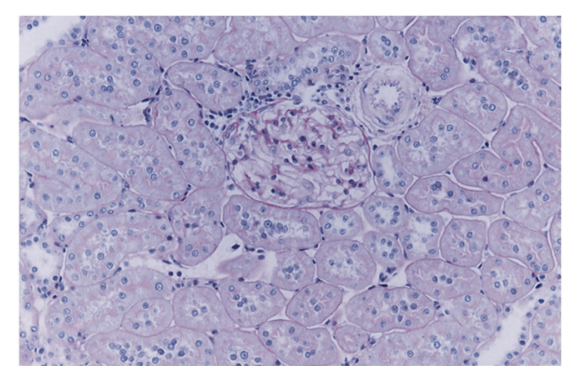

\section{Figure 7}

(a) Proteinuria following renal transplantation of Buffalo/Mna kidneys placed into nonproteinuric binephrectomized LEW. $1 \mathrm{~W}$ recipients treated with DST before transplantation to induce tolerance (two individuals). Comparison is made with proteinuria of agematched, unmodified, or uninephrectomized Buffalo/Mna rats $(n=5)$. Proteinuria is expressed as the ratio of the urinary protein to urinary creatinine concentration. Error bars indicate standard deviation. (b) Light microscope examination of Buffalo/Mna kidneys grafted into nonproteinuric LEW.1W rats (PAS staining, $\times 400$ ) showing a subnormal histology.

Buffalo/Mna rats, focal IgM deposits were observed on fibrotic areas that may have been related to the Buffalo/Mna disease (data not shown).

\section{Discussion}

In this article, we have revealed several lines of evidence suggesting that the spontaneous albuminuria developed by Buffalo/Mna can relapse in normal kidneys following transplantation. This observation may represent an original animal model of relapsing kidney disease after transplantation. The ethiopathogenesis of human idiopathic nephrotic syndrome and focal and segmental glomerular sclerosis remains unknown. Experimental FSGS has been induced by different means, mainly using chemical treatments and/or nephrotic reduction (15-19), but spontaneous FSGS that convincingly mimics the human primitive glomerular disease has been reported rarely (27-28).

The Buffalo/Mna rats were first reported because of the presence of a spontaneous thymoma (29) regulated by an autosomal dominant gene and associated with muscular weakness. Recently, this muscular disease has been demonstrated to be linked to plasmatic antiryanodine receptor Ab's (30). Other publications have focused on the Buffalo/Mna renal disease $(20,31)$. In these animals a selective proteinuria, which is permanently observed after 10 weeks, increases to high levels and reaches a plateau after 20 weeks of life. These rats also develop a nephrotic syndrome with hypoprotidemia and hyperlipidemia and are normotensive with a normal renal function and a normal histology at 1 month of age (20). Morphological changes in the glomeruli appear in 2 -month-old rats. At 4 months, eosinophilic tubular casts and, occasionally, FSGS lesions occur. However, at 6 months, all animals exhibit at least a few sclerotic lesions. Between $8.2 \%$ and $23.9 \%$ of segmental or global sclerosis is observed after 12 months and $37.8-52.1 \%$ at 22 months (20). Moreover, proteinuria has been shown to be functionally unrelated to the thymic disease (32). In addition, neonatal thymectomy has no effect on proteinuria (32). Two autosomal recessive genes were found to determine the susceptibility to glomerular sclerotic lesions by analysis of genetic segregation in animals derived from crossing Buffalo/Mna with other rat strains (31). Both genes are located on chromosome 13, which is also known to carry one of four genes appearing to control thymus enlargement, but different from the proteinuria gene Pur 1 . This chromosome localization corresponds to the long arm of the human chromosome 1 where the NPHS2 gene coding for podocin is localized (33-35). Therefore, we cannot exclude the possibility that Pur 1 is a homologue of the NPHS2 gene.

In humans, some types of FSGS have been found to be related to a genetic disorder and are usually corticoresistant. Familial FSGS is linked to genetic abnormalities on chromosome 1 , but usually does not recur after transplantation (34). The congenital nephrotic syndrome of the Finnish type is a rare disease in pediatric nephrology (36). In this case, the genetic abnormality located on chromosome 19 gives rise to a new transmembranous protein, nephrin, which is specifically expressed on the podocyte (37). Approximately $20 \%$ of these young patients develop a post-transplant nephrosis from the first day after transplantation (38) to 33 months (39). A recent publication suggests a role for an immunization against the normal nephrin protein presented by the graft in these patients (40).

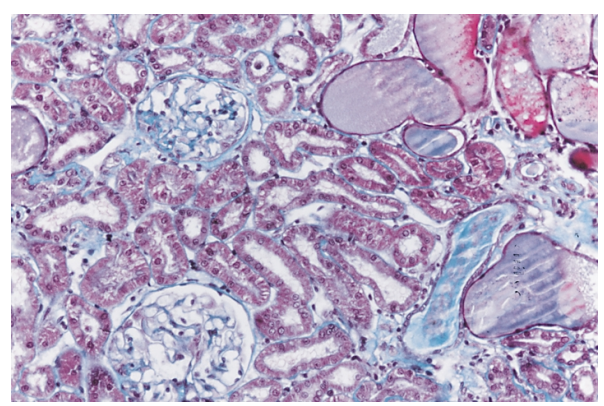

\section{Figure 8}

Light microscopic examination of native kidneys from 9-month-old Buffalo/Mna rats (PAS staining, $\times 200$ ) showing major tubular alterations and FSGS lesions. 
Clinically, the most informative observation suggesting the involvement of (an) albuminuric factor(s) in FSGS is the recurrence of albuminuria after renal transplantation (from a few hours to a few days later) in $30 \%$ of patients with primary FSGS. This elusive factor, which alters permselectivity and induces some architectural changes of the podocyte, may play a role in the first step of the disease, resulting in podocyte detachment and, subsequently, FSGS. When applied immediately following the recurrence after transplantation, and probably because of a recent and still reversible alteration in podocytes, plasma exchange (4-6) or immunoadsorption (7-8) can induce a transient remission of the disease. Because chronic plasmatic exchange or immunoadsorption are difficult to carry out in rats, and because the transfer of plasma from Buffalo/Mna to nonproteinuric rats does not trigger proteinuria (data not shown), we transplanted normal kidneys into binephrectomized proteinuric Buffalo/Mna recipients.

Transplantation of a normal kidney into an albuminuric recipient is more relevant to the human disease model and, in contrast to plasmatic transfer experiments, allows a prolonged exposure of kidney tissues to a putative circulating factor. However, this procedure could also induce weak allogeneic responses that could induce proteinuria. Since classical immunosuppressive regimens can interfere with the evolution of the disease, we took the option of removing all immunosuppressive drugs from the post-transplantation treatment regimen of the Buffalo/Mna recipients. We first transplanted Buffalo/Mna rats without any immunosuppressive treatment. In this condition, the proteinuria occurred 10 days after transplantation in three out of four rats; the relapse was rapid but not immediate, and this phenomenon is also observed in certain cases of human FSGS relapse (41). However, at sacrifice, histological examination demonstrated some tubulointerstitial lesions with an interstitial infiltrate that did not completely eliminate the possibility of chronic rejection.

Due to these ambiguities, a tolerant state for donor determinants was induced using donor-specific blood transfusion, which is one of the most potent procedures for inducing permanent (>100 days) tolerance of vascularized organs in adult rodent recipients (24). We have also demonstrated this long-term tolerance with the indefinite survival (>120 days) of LEW.1W hearts transplanted into Buffalo/Mna rats, without any histological signs of chronic rejection (data not shown). In DST-treated animals, proteinuria appeared 20 days after the transplantation, which is later than in those receiving no DST. The absence of proteinuria and of histological lesions in LEW.1W recipients of Wistar Furth kidneys following DST suggests that the transplantation procedure was not instrumental in the lesions observed in the Buffalo/Mna and favors the hypothesis of recurrence. There was no histological evidence of chronic rejection of the kidney grafts, where- as severe glomerular injury was observed, including FSGS lesions and some mesangiolytic areas noted occasionally. In these glomeruli, only slight and nonspecific IgM deposits were found in the mesangial space and in the areas of segmental sclerosis, as reported for the initial Buffalo/Mna disease (21). The tubulointerstitial lesions observed are probably the consequence of glomerular alterations, and the histological pattern of the LEW.1W kidneys grafted into Buffalo/Mna animals is similar to that observed in old Buffalo/Mna native kidneys (>1 year; Figure 8 ), again suggesting a recurrence of the initial disease in normal kidney tissue.

The immunological mechanisms underlying the effect of DST are still not well understood, but the role of a combined defect in the production of Th1 and Th2 cytokines (42-43) and an early and strong expression of TGF- $\beta$ has been demonstrated $(25,44)$. In our experiment, DST and its concomitant shift in the transcription of cytokines could have interfered with disease recurrence and thus explain the difference in the delay of proteinuria onset observed after the DST protocol.

We have observed that in the LEW.1W to Buffalo/Mna combination, the recipient's serum creatinine increased more than in the other groups. This observation is reminiscent of what has also been observed in the clinic where graft function is more frequently delayed in patients with relapses of FSGS (45), and this could correspond to an immediate aggression of the kidney by a putative albuminuric factor.

More importantly, when Buffalo/Mna kidneys were grafted into LEW.1W recipients, a significant decrease, followed by a stabilization of the protein excretion, was observed, while renal function remained stable, suggesting the reversibility of the lesions when Buffalo/Mna kidneys are transplanted into a normal environment. Such an observation was also reported in humans (46-47), but in this situation the role of the immunosuppressive treatment could not be excluded totally in the regression of the proteinuria. The absence of drugs in our model, except DST, which does not have any effect on Buffalo/Mna proteinuria (data not shown), reinforces the hypothesis of the role of a plasmatic factor in the alteration of glomerular permeability in these rats. Because the Buffalo/Mna kidney disease seems to be due to a genetic disorder, we suspect a functional and structural abnormality of a protein specific to the podocyte, as recently found in humans. Nevertheless, we were unable to identify any Ig deposition in the LEW.1W transplants (as described in humans). In addition, the remission of the proteinuria and lesions in the Buffalo/Mna kidney to LEW.1W combination is suggestive of the presence of an extrarenal permeability factor.

Despite some discrepancies with the human disease, such as the delay of the recurrence of proteinuria and tubular alterations, this model of relapsing nephrotic syndrome after transplantation may help in determining the mechanisms involved in INS/FSGS glomerular injury in humans. 


\section{Acknowledgments}

This work was supported by the Fondation Transvie. We thank Joanna Ashton for editing the manuscript.

1. Korbet, S.M. 1995. Management of idiopathic nephrosis in adults, including steroid-resistant nephrosis. Curr. Opin. Nephrol. Hypertens. 4:169-176.

2. D'Agati, V. 1994. The many masks of focal segmental glomerulosclerosis. Kidney Int. 46:1223-1241.

3. Dantal, J., Giral, M., Hoormant, M., and Soulillou, J.P. 1995. Glomerulonephritis recurrences after kidney transplantation. Curr. Opin. Nephrol. Hypertens. 4:146-154.

4. Dantal, J., et al. 1991. Recurrent nephrotic syndrome following renal transplantation in patients with focal glomerulosclerosis. A one-center study of plasma exchange effects. Transplantation. 52:827-831.

5. Cochat, P., et al. 1993. Recurrent nephrotic syndrome after transplantation: early treatment with plasmaphaeresis and cyclophosphamide. Pediatr. Nephrol. 7:50-54.

6. Artero, M.L., Sharma, R., Savin, V.J., and Vincenti, F. 1994. Plasmapheresis reduces proteinuria and serum capacity to injure glomeruli in patients with recurrent focal glomerulosclerosis. Am. J. Kidney Dis. 23:574-581.

7. Dantal, J., et al. 1994. Effect of plasma protein adsorption on protein excretion in kidney-transplant recipients with recurrent nephrotic syndrome. N. Engl. J. Med. 330:7-14.

8. Dantal, J., et al. 1998. Antihuman immunoglobulin affinity immunoadsorption strongly decreases proteinuria in patients with relapsing nephrotic syndrome. J. Am. Soc. Nephrol. 9:1709-1715.

9. Savin, V.J., et al. 1996. Circulating factor associated with increased glomerular permeability to albumin in recurrent focal segmental glomerulosclerosis. N. Engl.J. Med. 334:878-883.

10. Godfrin, Y., Dantal, J., Bouhours, J.F., Heslan, J.M., and Soulillou, J.P. 1996 A new method of measuring albumin permeability in isolated glomeruli. Kidney Int. 50:1352-1357.

11. Garin, E.H., and Corontzes, N. 1992. Effect of lymphokine from nephrotic peripheral blood mononuclear cells on catabolism of rat glomerular basement membrane sulfated compounds. Nephron. 62:416-421.

12. Le Berre, L., et al. 2000. Effect of plasma fractions from patients with focal and segmental glomerulosclerosis on rat proteinuria. Kidney Int. 58:2502-2511

13. Sharma, M., Sharma, R., McCarthy, E.T., and Savin, V.J. 1999. "The FSGS factor"; enrichment and in vivo effect of activity from focal segmental glomerulosclerosis plasma. J. Am. Soc. Nephrol. 10:552-561.

14. Kuijpers, M.H., Provoost, A.P., and de Jong, W. 1986. Development of hypertension and proteinuria with age in fawn-hooded rats. Clin. Exp. Pharmacol. Physiol. 13:201-209.

15. Olivier, J., et al. 1994. Proteinuria and impaired glomerular permselectivity in uninephrectomized fawn-hooded rats. Am.J. Physiol. 267:F917-F925.

16. van Goor, H., Fidler, V., Weening, J.J., and Grond, J. 1991. Determinants of focal and segmental glomerulosclerosis in the rat after renal ablation. Evidence for involvement of macrophages and lipids. Lab. Invest. 64:754-765.

17. Bertani, T., et al. 1982. Adriamycin-induced nephrotic syndrome in rats: sequence of pathologic events. Lab. Invest. 46:16-23.

18. Whiteside, C.I., Cameron, R., Munk, S., and Levy, J. 1993. Podocytic cytoskeletal disaggregation and basement-membrane detachment in puromycin aminonucleoside nephrosis. Am. J. Pathol. 142:1641-1653.

19. Ginevri, F., et al. 1993. Progression of chronic adriamycin nephropathy in leukopenic rats. Nephron. 63:79-88.

20. Kato, F., Watanabe, M., and Matsuyama, M.. 1983. Nephrotic syndrome in spontaneous thymoma rats, Buffalo/Mna. Biomedical Research. 4:105-110.

21. Nakamura, T., et al. 1986. Sclerotic lesions in the glomeruli of Buffalo/Mna rats. Nephron. 43:50-55.

22. Engelbrecht, G., Kahn, D., Duminy, M., and Hickman, R. 1992. New rapid technique for renal transplantation in the rat. Microsurgery. 13:340-344.

23. Soulillou, J.P., Blandin, F., Gunther, E., and Lemoine, V. 1984. Genetics of the blood transfusion effect on heart allografts in rats. Transplantation. 38:63-67.
24. Josien, R., Heslan, M., Brouard, S., Soulillou, J.P., and Cuturi, M.C. 1998. Critical requirement for graft passenger leukocytes in allograft tolerance induced by donor blood transfusion. Blood. 92:4539-4544.

25. Josien, R., et al. 1998. A critical role for transforming growth factor-beta in donor transfusion-induced allograft tolerance. J. Clin. Invest. 102:1920-1926

26. Solez, K., et al. 1993. International standardization of criteria for the histologic diagnosis of renal allograft rejection: the Banff working classification of kidney transplant pathology. Kidney Int. 44:411-422.

27. Nishimura, M., et al. 1994. Focal segmental glomerular sclerosis, a type of intractable chronic glomerulonephritis, is a stem cell disorder. J. Exp. Med. 179:1053-1058.

28. Abramowsky, C.R., Aikawa, M., Swinehart, G.L., and Snajdar, R.M. 1984 Spontaneous nephrotic syndrome in a genetic rat model. Am. J. Pathol. 117:400-408.

29. Matsuyama, M., Yamada, C., and Hiai, H. 1986. A single dominant susceptible gene determines spontaneous development of thymoma in BUF/Mna rat. JPN. J. Cancer Res. 77:1066-1068.

30. Iwasa, K., Komai, K., and Takamori, M. 1998. Spontaneous thymoma rat as a model for myasthenic weakness caused by anti-ryanodine receptor antibodies. Muscle Nerve. 21:1655-1660.

31. Matsuyama, M., et al. 1990. Genetic regulation of the development of glomerular sclerotic lesions in the BUF/Mna rat. Nephron. 54:334-337.

32. Nakamura, T., et al. 1988. The effect of thymectomy on the development of nephropathy in spontaneous thymoma rats of the BUF/Mna strain. Clin. Exp. Immunol. 71:350-352.

33. Murayama, S., et al. 1998. A genetic locus susceptible to the overt proteinuria in BUF/Mna rat. Mamm. Genome. 9:886-888.

34. Fuchshuber, A., et al. 1995. Mapping a gene (SRN1) to chromosome 1q25 q31 in idiopathic nephrotic syndrome confirms a distinct entity of autosomal recessive nephrosis. Hum. Mol. Genet. 4:2155-2158.

35. Boute, N., et al. 2000. NPHS2, encoding the glomerular protein podocin, is mutated in autosomal recessive steroid-resistant nephrotic syndrome. Nat. Genet. 24:349-354.

36. Holmberg, C., et al. 1995. Management of congenital nephrotic syndrome of the Finnish type. Pediatr. Nephrol. 9:87-93.

37. Kestila, M., et al. 1998. Positionally cloned gene for a novel glomerular protein-nephrin-is mutated in congenital nephrotic syndrome. Mol. Cell. 1:575-582.

38. Barayan, S., et al. 2001. Immediate post-transplant nephrosis in a patient with congenital nephrotic syndrome. Pediatr. Nephrol. 16:547-549.

39. Laine, J., et al. 1993. Post-transplantation nephrosis in congenital nephrotic syndrome of the Finnish type. Kidney Int. 44:867-874.

40. Wang, S., et al. 2001. Recurrence of nephrotic syndrome after transplantation in CNF is due to autoantibodies to nephrin. Exp. Nephrol. 9:327-331

41. Senggutuvan, P., et al. 1990. Recurrence of focal segmental glomerulosclerosis in transplanted kidneys: analysis of incidence and risk factors in 59 allografts. Pediatr. Nephrol. 4:21-28.

42. Flye, M.W., et al. 1995. Donor-specific transfusions have long-term beneficial effects for human renal allografts. Transplantation. 60:1395-1401.

43. Dallman, M.J., Shiho, O., Page, T.H., Wood, K.J., and Morris, P.J. 1991. Peripheral tolerance to alloantigen results from altered regulation of the interleukin 2 pathway. J. Exp. Med. 173:79-87.

44. Bugeon, L., et al. 1992. Peripheral tolerance of an allograft in adult rats characterization by low interleukin- 2 and interferon-gamma mRNA levels and by strong accumulation of major histocompatibility complex transcripts in the graft. Transplantation. 54:219-225.

45. Kim, E., et al. 1994. Recurrence of steroid-resistant nephrotic syndrome in kidney transplants is associated with increased acute renal failure and acute rejection. Kidney Int. 45:1440-1445.

46. Ali, A.A., et al. 1994. Minimal-change glomerular nephritis. Normal kidneys in an abnormal environment? Transplantation. 58:849-852.

47. Rea, R., Smith, C., Sandhu, K., Kwan, J., and Tomson, C. 2001. Successful transplant of a kidney with focal segmental glomerulosclerosis. Nephrol. Dial. Transplant. 16:416-417. 